\title{
On the solution of an acoustic wave equation with variable-order derivative loss operator
}

Abdon Atangana*

"Correspondence:

abdonatangana@yahoo.fr

Institute for Groundwater Studies,

Faculty of Natural and Agricultural

Sciences, University of the Free

State, Bloemfontein, 9300, South

Africa

\begin{abstract}
When modeling sound propagation, the use of fractional derivatives leads to models that better describe observations of attenuation and dispersion. The wave equation for viscous losses involving integer-order derivatives only leads to an attenuation which is proportional to the square of the frequency. This does not always reflect reality. The acoustic wave equation with loss operator is generalized to the concept of variable-order derivatives in this work. The generalized equation is solved via the Crank-Nicholson scheme. The stability and the convergence of this case are examined in detail.
\end{abstract}

Keywords: acoustic wave equation; loss operator; variable-order derivative; Crank-Nicholson scheme; stability; convergence

\section{Introduction}

Fractional derivatives are well suited to describe wave propagation in complex media. When introduced in classical wave equations, they allow the modeling of attenuation and dispersion that better describes sound propagation in biological tissues. Fractional derivatives have been used for modeling heat transfer or diffusion [1, 2], seismic data [3] and sound wave propagation, [4-6] only to name a few. When modeling sound propagation, the use of fractional derivatives leads to models that better describe observations of attenuation and dispersion [7]. The wave equation for viscous losses involving integer-order derivatives only leads to an attenuation which is proportional to the square of the frequency. This does not always reflect reality. For instance, for biological tissues [8] and marine sediments [9], the frequency dependency of attenuation and dispersion is more complicated. Different forms of the wave equation have been proposed to reflect this complexity $[4,7,10-12]$.

Several simulators take a modified nonlinear wave equation as a starting point by replacing the traditional loss operator by fractional derivatives $[7,13,14]$ or a convolution in time $[15,16]$. Their justification for modifying the standard wave equations is the ability of fractional derivatives to lead to a dispersion equation that better describes attenuation and dispersion. A wave equation based on fractional constitutive equations gives an alternative to modeling absorption and dispersion in complex media like biological tissues. However, in the case where the medium, through which these sounds are propagating, is variable or heterogeneous, neither the wave equation described by integer order nor that described by constant fractional order are suited for describing the phenomena. To solve the above problems, the variable-order ( $\mathrm{VO})$ fractional acoustic wave equation models are 
suggested for use in this work. This present work is therefore devoted to the discussion underpinning the description of the extension of an acoustic wave equation to the concept of the variational-order derivative and the solution of the generalized equation using the Crank-Nicholson scheme.

\section{A possible modification of an acoustic wave equation}

For the readers that are not acquainted with the concept of the variational-order derivative, we start this section by presenting the basic definition of this derivative.

\subsection{Variable-order differential operator}

Let $f: \mathbb{R} \rightarrow \mathbb{R}, x \rightarrow f(x)$ denote a continuous but necessary differentiable, let $\alpha(x)$ be a continuous function in $(0,1]$. Then its variable-order differential in $[a, \infty)$ is defined as

$$
D_{0}^{\alpha(x)}(f(x))=\frac{1}{\Gamma(1-\alpha(x))} \int_{0}^{x}(x-t)^{-\alpha(t)} \frac{d f(t)}{d t} d t .
$$

The above derivative is called the Caputo variational-order differential operator, in addition, the derivative of the constant is zero.

\subsection{Statement of the problem}

Attenuation of propagating waves in many materials follows a power law over several decades of frequency variation [17], Szabo and Wu [18]

$$
\alpha=\alpha_{0} \omega^{y},
$$

where $\alpha_{0}$ and $y$ are constants that characterize the medium. Such attenuation can be described by wave equations with particular loss operators.

In this paper we consider only the acoustic wave equation with loss operator. A wave equation with a loss operator $L$, which in the general case is a convolution, is

$$
\nabla^{2} u-\frac{1}{c_{0}^{2}} \frac{\partial^{2} u}{\partial t^{2}}+L u=0
$$

where $c_{0}$ is a propagation velocity and $u(x ; y ; z ; t)$ is the particle displacement. As is well established, the form of the loss operator is simple to justify from the underlying physics for only a few values of $y$. One such case is classical visco-elasticity, which is used as a first-order model, for instance, air and water. The loss operator is then [17]

$$
L u=\tau \frac{\partial\left(\nabla^{2} u\right)}{\partial t} .
$$

It gives a power law with exponent $y=2$ when $\omega \tau \ll 1$, where $\tau$ is medium-specific relaxation time. In 1994 Szabo developed a wave equation for $y \in[0 ; 2]$ which for $y=2$ was similar to an approximation to equation (2.3) where the viscoelastic loss term instead is a third-order time derivative [18]

$$
L u=\left(\frac{\tau}{c_{0}^{2}}\right) \frac{\partial^{3} u}{\partial t^{3}} .
$$


In 2004 Chen and Holm reformulated the lossy wave equation in terms of a spatial fractional derivative with a fractional Laplacian. Under these circumstances, the loss operator was proposed as [19]

$$
L u \propto-\frac{\partial\left(-\nabla^{2}\right)^{y / 2} u}{\partial t} .
$$

This operator covers exactly the loss operator of equation (2.3) for $y=2$, not just its lowfrequency approximation. However, a disadvantage of these formulations is that they do not guarantee a causal solution. Recently Wismer [20] proposed a loss operator to be in the form of

$$
L u=\tau^{y-1} \frac{\partial^{y-1}\left(\nabla^{2} u\right)}{\partial t^{y-1}} .
$$

The above proposed loss operator is equivalent to the loss operator of the viscoelastic equation for $y=2$. This operator is interesting as it turns out to be based on an underlying fractional Kelvin-Voigt model despite the impression Wismer [20] gives that it was found by inspection just like the previous operators. Although the wave equations given here may successfully be applied for wave propagation simulation, most of them are nevertheless derived through ad hoc procedures which are not directly linked to more basic physical principles.

In this paper, we propose the loss operator to be converted to the concept of variationalorder derivative as follows:

$$
L u=\tau \frac{\partial^{\rho(x, t)}(u)}{\partial t^{\rho(x, t)}},
$$

where $\rho(x, t)$ is a smooth function with the range $(1,2]$ and $\frac{\partial^{\rho(x, t)}}{\partial t^{\rho(x, t)}}$ is the variational-order derivative. Therefore equation (2.2) can then be reformulated as follows:

$$
\nabla^{2} u-\frac{1}{c_{0}^{2}} \frac{\partial^{2} u}{\partial t^{2}}+\tau \frac{\partial^{\rho(x, t)}(u)}{\partial t^{\rho(x, t)}}=0
$$

The above equation is then called the 'variable-order derivative acoustic wave equation with loss operator.' This new equation does not have obviously an exact analytical solution. In particular, the equation cannot be solved analytically. It is therefore important to examine its solution numerically. The aim of the next section is then devoted to the discussion underpinning the numerical solution of the modified equation using the Crank-Nicholson scheme.

\section{Numerical solution of a variable-order derivative acoustic wave equation with loss operator}

Numerical methods yield approximate solutions to the governing equation through the discretization of space and time. Within the discredited problem domain, the variable internal properties, boundaries and stresses of the system are approximated. Deterministic, distributed-parameter, numerical models can relax the rigid idealized conditions of analytical models or lumped-parameter models, and they can therefore be more realistic 
and flexible for simulating fields conditions. The finite difference schemes for constantorder time or space fractional diffusion equations have been widely studied [21, 22]. For constant-order time fractional diffusion equations, the implicit difference approximation scheme was proposed in [23]. The weighted average finite difference methods were introduced in [24]. The matrix approach for fractional diffusion equations was proposed in [25], and Hanert proposed a flexible numerical scheme for the discretization of the spacetime fractional diffusion equation [26]. Lately, the numerical schemes for a VO space fractional advection-dispersion equation was considered by the author of [27]. An investigation of the explicit scheme for a VO nonlinear space fractional diffusion equation was done in [28]. Before performing the numerical methods, we assume that equation (2.3) has a unique and sufficiently smooth solution. To establish the numerical schemes for the above equation, we let $x_{l}=l h, 0 \leq l \leq M, M h=L, t_{k}=k \sigma, 0 \leq k \leq N, N \sigma=T, h$ is the step and $\tau$ is the time size, $M$ and $N$ are grid points.

\subsection{Crank-Nicholson scheme [29]}

We introduce the Crank-Nicholson scheme as follows. Firstly, the discretization of firstand second-order space derivative is stated as follows:

$$
\begin{aligned}
& \frac{\partial u}{\partial x}=\frac{1}{2}\left(\left(\frac{u\left(x_{l+1}, t_{k+1}\right)-u\left(x_{l-1}, t_{k+1}\right)}{2(h)}\right)+\left(\frac{u\left(x_{l+1}, t_{k}\right)-\Phi\left(x_{l-1}, t_{k}\right)}{2(h)}\right)\right)+O(h), \\
& \frac{\partial^{2} u\left(x_{l}, t_{k+1}\right)}{\partial t^{2}}=\frac{1}{2}\left(\frac{\left(u\left(x_{l+1}, t_{k+1}\right)-2 u\left(x_{l+1}, t_{k}\right)+u\left(x_{l+1}, t_{k-1}\right)\right)}{h^{2}}\right. \\
& \left.+\frac{\left(u\left(x_{l}, t_{k+1}\right)-2 u\left(x_{l}, t_{k}\right)+u\left(x_{l}, t_{k-1}\right)\right)}{h^{2}}\right)+O\left(h^{2}\right), \\
& \frac{\partial^{2} u}{\partial x^{2}}=\frac{1}{2}\left(\left(\frac{u\left(x_{l+1}, t_{k+1}\right)-2 u\left(x_{l}, t_{k+1}\right)+u\left(x_{l-1}, t_{k+1}\right)}{(h)^{2}}\right)\right. \\
& \left.+\left(\frac{u\left(x_{l+1}, t_{k}\right)-2 u\left(x_{l}, t_{k}\right)+u\left(x_{l-1}, t_{k}\right)}{(h)^{2}}\right)\right)+O\left(h^{2}\right), \\
& u=\frac{1}{2}\left(u\left(x_{l}, t_{k+1}\right)+u\left(x_{l}, t_{k}\right)\right) \text {. }
\end{aligned}
$$

The Crank-Nicholson scheme for the VO time fractional diffusion model can be stated as follows:

$$
\begin{aligned}
\frac{\partial^{\rho_{l}^{k+1}} u\left(x_{l}, t_{k+1}\right)}{\partial t^{\rho_{l}^{k+1}}}= & \frac{\sigma^{-\rho_{l}^{k+1}}}{\Gamma\left(2-\rho_{l}^{k+1}\right)}\left(u\left(x_{l}, t_{k+1}\right)-u\left(x_{l}, t_{k}\right)\right. \\
& \left.+\sum_{j=1}^{k}\left[u\left(x_{l}, t_{k+1-j}\right)-u\left(x_{l}, t_{k-j}\right)\right]\left[(j+1)^{1-\rho_{l}^{k+1}}-(j)^{1-\rho_{l}^{k+1}}\right]\right) .
\end{aligned}
$$

Now, replacing equations (3.1), (3.2), (3.3) and (3.4) in (2.3), we obtain the following:

$$
\begin{aligned}
& \frac{\tau \sigma^{-\rho_{l}^{k+1}}}{\Gamma\left(2-\rho_{l}^{k+1}\right)}\left(u\left(x_{l}, t_{k+1}\right)-u\left(x_{l}, t_{k}\right)\right. \\
& \left.\quad+\sum_{j=1}^{k}\left[u\left(x_{l}, t_{k+1-j}\right)-u\left(x_{l}, t_{k-j}\right)\right]\left[(j+1)^{1-\rho_{l}^{k+1}}-(j)^{1-\rho_{l}^{k+1}}\right]\right)
\end{aligned}
$$




$$
\begin{aligned}
& +\frac{1}{2}\left(\left(\frac{u\left(x_{l+1}, t_{k+1}\right)-2 u\left(x_{l}, t_{k+1}\right)+u\left(x_{l-1}, t_{k+1}\right)}{(h)^{2}}\right)\right. \\
& \left.+\left(\frac{u\left(x_{l+1}, t_{k}\right)-2 u\left(x_{l}, t_{k}\right)+u\left(x_{l-1}, t_{k}\right)}{(h)^{2}}\right)\right) \\
& =-\frac{1}{c_{0}^{2}} \frac{1}{2}\left(\left(\frac{u\left(x_{l+1}, t_{k+1}\right)-2 u\left(x_{l+1}, t_{k}\right)+u\left(x_{l+1}, t_{k-1}\right)}{(h)^{2}}\right)\right. \\
& \left.+\left(\frac{u\left(x_{l}, t_{k+1}\right)-2 u\left(x_{l}, t_{k}\right)+u\left(x_{l}, t_{k-1}\right)}{(h)^{2}}\right)\right) .
\end{aligned}
$$

For simplicity, let us put

$$
\begin{aligned}
& b_{j}^{l, k+1}=(j+1)^{1-\alpha_{l}^{k+1}}-(j)^{1-\alpha_{l}^{k+1}} ; \quad T_{l}^{k+1}=-\frac{\Gamma\left(2-\rho_{l}^{k+1}\right) \sigma^{\rho_{l}^{k+1}}}{2 \tau c_{0}^{2} h^{2}} \text { and } \\
& \lambda_{j}^{l, k+1}=b_{j-1}^{l, k+1}-b_{j}^{l, k+1} \quad \text { and } u\left(x_{l}, t_{k}\right)=u_{l}^{k} .
\end{aligned}
$$

Equation (3.5) becomes

$$
\begin{aligned}
& u_{l}^{k+1}\left(1+2 T_{l}^{k+1}-c_{0}^{2} T_{l}^{k+1}\right) \\
& =u_{l+1}^{k+1}\left(-c_{0}^{2} T_{l}^{k+1}-T_{l}^{k+1}\right)+u_{l-1}^{k+1}\left(-c_{0}^{2} T_{l}^{k+1}-T_{l}^{k+1}\right) \\
& \quad+u_{l}^{k}\left(1+c_{0}^{2} T_{l}^{k+1}+2 T_{l}^{k+1}\right)+u_{l}^{k-1}\left(-T_{l}^{k+1}\right)-\sum_{j=1}^{k}\left[u_{l}^{k+1-j}-u_{l}^{k-j}\right] b_{j}^{l, k+1} .
\end{aligned}
$$

\section{Stability analysis of the Crank-Nicholson scheme}

In this section, we analyze the stability conditions of the Crank-Nicholson scheme for the generalized acoustic wave equation.

Let $\zeta_{l}^{k}=u_{l}^{k}-U_{l}^{k}$, here $U_{l}^{k}$ is the approximate solution at the point $\left(x_{l}, t_{k}\right)(k=1,2, \ldots, N$, $l=1,2, \ldots, M-1)$ and, in addition, $\zeta^{k}=\left[\zeta_{1}^{k}, \zeta_{2}^{k}, \ldots, \zeta_{M-1}^{k}\right]^{T}$ and the function $\zeta^{k}(x)$ is chosen to be

$$
\zeta^{k}(x)= \begin{cases}\zeta_{l}^{k} & \text { if } x_{l}-\frac{h}{2}<x \leq x_{l}+\frac{h}{2}, l=1,2, \ldots, M-1 \\ 0 & \text { if } L-\frac{h}{2}<x \leq L\end{cases}
$$

Then the function $\zeta^{k}(x)$ can be expressed in Fourier series as follows:

$$
\begin{aligned}
& \zeta^{k}(x)=\sum_{m=-\infty}^{m=\infty} \delta_{m}(m) \exp [2 i \pi m k / L] \\
& \delta_{k}(x)=\frac{1}{L} \int_{0}^{L} \rho^{k}(x) \exp \left[\frac{2 i \pi m x}{L}\right] d x
\end{aligned}
$$

It was established by [30] that

$$
\left\|\rho^{2}\right\|_{2}^{2}=\sum_{m=-\infty}^{m=\infty}\left\|\delta_{k}(m)\right\|^{2}
$$


Observe that for all $k, l \geq 1,0 \leq 1-\alpha_{l}^{k+1}<1$. Then the following properties of the coefficients $T_{l}^{k+1}, \lambda_{j}^{l, k+1}$ and $b_{l}^{k+1}$ can be established.

(1) $T_{l}^{k+1}$ are negative for all $l=1,2, \ldots, M-1$.

(2) $0<\lambda_{j}^{l, k} \leq \lambda_{j-1}^{l, k} \leq 1 \quad$ for all $l=1,2, \ldots, M-1$.

(3) $\quad 0 \leq b_{j}^{l, k} \leq 1, \quad \sum_{j=0}^{k-1} b_{j+1}^{l, k+1}=1-\lambda_{k}^{l, k+1} \quad$ for all $l=1,2, \ldots, M-1$.

If we assume that $\zeta_{l}^{k}$ in equation (4.1) can be put in the delta-exponential form as follows:

$$
\zeta_{l}^{k}=\delta_{k} \exp [i \varphi l k]
$$

where $\varphi$ is a real spatial wave number, now replacing the above equation (4.5) in (3.6) we obtain

$$
\begin{aligned}
& \delta_{1}\left(1+4 \sin ^{2}\left(\frac{\varphi h}{2}\right) T_{l}^{1}-c_{0}^{2} \sin ^{2}\left(\frac{\varphi h}{2}\right) T_{l}^{1}\right) \\
&= \delta_{0}\left(1+c_{0}^{2} T_{l}^{1} \sin ^{2}\left(\frac{\varphi h}{2}\right)+4 T_{l}^{1} \sin ^{2}\left(\frac{\varphi h}{2}\right)\right) ; \quad k=0, \\
& \delta_{k+1}\left(1+4 \sin ^{2}\left(\frac{\varphi h}{2}\right) T_{l}^{k+1}-2 c_{0}^{2} T_{l}^{k+1} \sin ^{2}\left(\frac{\varphi h}{2}\right)\right) \\
&=+\delta_{k}\left(1+c_{0}^{2} T_{l}^{k+1} \sin ^{2}\left(\frac{\varphi h}{2}\right)+2 T_{l}^{k+1} \sin ^{2}\left(\frac{\varphi h}{2}\right)-\lambda_{1}^{l, k+1}\right) \\
& \quad-\sum_{j=0}^{k-1} \lambda_{j+1}^{l, k+1} \delta_{k-j}+\lambda_{k}^{l, k+1} \delta_{0} \quad \text { for } k \geq 1 .
\end{aligned}
$$

Now, according to (1), $T_{l}^{k+1}$ are negative for all $l=1,2 \ldots, M-1$. Then from above equation (4.6), we have the following

$$
\begin{aligned}
& \delta_{1}=+\delta_{0} \frac{\left(1+c_{0}^{2} T_{l}^{1} \sin ^{2}\left(\frac{\varphi h}{2}\right)+4 T_{l}^{1} \sin ^{2}\left(\frac{\varphi h}{2}\right)\right)}{\left(1+4 \sin ^{2}\left(\frac{\varphi h}{2}\right) T_{l}^{1}-c_{0}^{2} \sin ^{2}\left(\frac{\varphi h}{2}\right) T_{l}^{1}\right)}, \\
& \delta_{k+1}=\frac{\delta_{k}\left(1+c_{0}^{2} T_{l}^{k+1} \sin ^{2}\left(\frac{\varphi h}{2}\right)+2 T_{l}^{k+1} \sin ^{2}\left(\frac{\varphi h}{2}\right)-\lambda_{1}^{l, k+1}\right)-\sum_{j=0}^{k-1} \lambda_{j+1}^{l, k+1} \delta_{k-j}+\lambda_{k}^{l, k+1} \delta_{0}}{\left(1+4 \sin ^{2}\left(\frac{\varphi h}{2}\right) T_{l}^{k+1}-2 c_{0}^{2} T_{l}^{k+1} \sin ^{2}\left(\frac{\varphi h}{2}\right)\right)}
\end{aligned}
$$

$$
\text { for } k \geq 1 \text {. }
$$

Our next investigation here is to show that for all $k=1,2, \ldots, N-1$, the solution of equation (4.7) satisfies the following condition:

$$
\left|\delta_{k}\right|<\left|\delta_{0}\right|
$$

To achieve this, we make use of the recurrence technique on the natural number $k$. 
For $k=1$ and remembering that $T_{l}^{k+1}$ are negative for all $l=1,2, \ldots, M-1$, then we obtain

$$
\frac{\left|\delta_{1}\right|}{\left|\delta_{0}\right|}=\left|\frac{\left(1+c_{0}^{2} T_{l}^{1} \sin ^{2}\left(\frac{\varphi h}{2}\right)+4 T_{l}^{1} \sin ^{2}\left(\frac{\varphi h}{2}\right)\right)}{\left(1+4 \sin ^{2}\left(\frac{\varphi h}{2}\right) T_{l}^{1}-c_{0}^{2} \sin ^{2}\left(\frac{\varphi h}{2}\right) T_{l}^{1}\right)}\right|<1
$$

Assume that for $m=2,3, \ldots, k$, the property is verified. Then

$$
\begin{aligned}
& \left|\delta_{k+1}\right| \\
& =\left|\frac{\left(1+c_{0}^{2} T_{l}^{k+1} \sin ^{2}\left(\frac{\varphi h}{2}\right)+2 T_{l}^{k+1} \sin ^{2}\left(\frac{\varphi h}{2}\right)-\lambda_{1}^{l, k+1}\right) \delta_{k}-\sum_{j=0}^{k-1} \lambda_{j+1}^{l, k+1} \delta_{k-j}+\lambda_{k}^{l, k+1} \delta_{0}}{\left(1+4 \sin ^{2}\left(\frac{\varphi h}{2}\right) T_{l}^{k+1}-2 c_{0}^{2} T_{l}^{k+1} \sin ^{2}\left(\frac{\varphi h}{2}\right)\right)}\right| .
\end{aligned}
$$

Making use of the triangular inequality, we obtain the following inequality:

$$
\begin{aligned}
\left|\delta_{k+1}\right| & \left(\left|\left(1+c_{0}^{2} T_{l}^{k+1} \sin ^{2}\left(\frac{\varphi h}{2}\right)+2 T_{l}^{k+1} \sin ^{2}\left(\frac{\varphi h}{2}\right)-\lambda_{1}^{l, k+1}\right)\right|\left|\delta_{k}\right|\right. \\
& \left.+\left|\sum_{j=0}^{k-1} \lambda_{j+1}^{l, k+1}\right|\left|\delta_{k-j}\right|+\left|\lambda_{k}^{l, k+1}\right|\left|\delta_{0}\right|\right) \\
& /\left|1+4 \sin ^{2}\left(\frac{\varphi h}{2}\right) T_{l}^{k+1}-2 c_{0}^{2} T_{l}^{k+1} \sin ^{2}\left(\frac{\varphi h}{2}\right)\right|
\end{aligned}
$$

Using the recurrence hypothesis, we have

$$
\begin{aligned}
& \left|\delta_{k+1}\right| \leq \frac{\left|\left(1+c_{0}^{2} T_{l}^{k+1} \sin ^{2}\left(\frac{\varphi h}{2}\right)+2 T_{l}^{k+1} \sin ^{2}\left(\frac{\varphi h}{2}\right)\right)\right|\left|\delta_{0}\right|+\left|\sum_{j=0}^{k-1} \lambda_{j+1}^{l, k+1}\right|\left|\delta_{0}\right|}{\left|1+4 \sin ^{2}\left(\frac{\varphi h}{2}\right) T_{l}^{k+1}-2 c_{0}^{2} T_{l}^{k+1} \sin ^{2}\left(\frac{\varphi h}{2}\right)\right|} \\
& \left|\delta_{k+1}\right| \leq \frac{\left|\left(1+c_{0}^{2} T_{l}^{k+1} \sin ^{2}\left(\frac{\varphi h}{2}\right)+2 T_{l}^{k+1} \sin ^{2}\left(\frac{\varphi h}{2}\right)\right)\right|\left|\delta_{0}\right|}{\left|1+4 \sin ^{2}\left(\frac{\varphi h}{2}\right) T_{l}^{k+1}-2 c_{0}^{2} T_{l}^{k+1} \sin ^{2}\left(\frac{\varphi h}{2}\right)\right|} \\
& \left|\delta_{k+1}\right| \leq \frac{\left|\left(1+c_{0}^{2} T_{l}^{k+1} \sin ^{2}\left(\frac{\varphi h}{2}\right)-2 T_{l}^{k+1} \sin ^{2}\left(\frac{\varphi h}{2}\right)\right)\right|\left|\delta_{0}\right|}{\left|1+4 \sin ^{2}\left(\frac{\varphi h}{2}\right) T_{l}^{k+1}-2 c_{0}^{2} T_{l}^{k+1} \sin ^{2}\left(\frac{\varphi h}{2}\right)\right|} \\
& \left|\delta_{k+1}\right| \leq\left|\delta_{0}\right|
\end{aligned}
$$

which completes the proof.

\section{Convergence analysis of the Crank-Nicholson scheme}

If we assume that $u\left(x_{l}, t_{k}\right)(l=1,2, \ldots, M, k=1,2, \ldots, N-1)$ is the exact solution of our problem at the point $\left(x_{l}, t_{k}\right)$, by letting $\Omega_{l}^{k}=u\left(x_{l}, t_{k}\right)-u_{l}^{k}$ and $\Omega^{k}=\left(0, \Omega_{1}^{k}, \Omega_{2}^{k}, \ldots, \Omega_{M-1}^{k}\right)$, substituting this in equation (3.6), we obtain

$$
\begin{aligned}
& \Omega_{l}^{1}\left(1+2 T_{l}^{1}-c_{0}^{2} T_{l}^{1}\right)+\Omega_{l+1}^{1}\left(c_{0}^{2} T_{l}^{1}+T_{l}^{1}\right)+\Omega_{l-1}^{1}\left(c_{0}^{2} T_{l}^{1}+T_{l}^{1}\right)=E_{l}^{1}, \\
& \Omega_{l}^{k+1}\left(1+2 T_{l}^{k+1}-c_{0}^{2} T_{l}^{k+1}\right)+\Omega_{l+1}^{k+1}\left(c_{0}^{2} T_{l}^{k+1}+T_{l}^{k+1}\right)+\Omega_{l-1}^{k+1}\left(c_{0}^{2} T_{l}^{k+1}+T_{l}^{k+1}\right) \\
& \quad=\sum_{j=1}^{k}\left[\Omega_{l}^{k+1-j}-\Omega_{l}^{k-j}\right] \lambda_{j}^{l, k+1}+E_{l}^{k+1}, \quad k \geq 1 .
\end{aligned}
$$


The expression $E_{l}^{k+1}$ is given in the following form:

$$
\begin{aligned}
& \frac{\tau \sigma^{-\rho_{l}^{k+1}}}{\Gamma\left(2-\rho_{l}^{k+1}\right)}\left(u\left(x_{l}, t_{k+1}\right)-u\left(x_{l}, t_{k}\right)+\sum_{j=1}^{k}\left[u\left(x_{l}, t_{k+1-j}\right)-u\left(x_{l}, t_{k-j}\right)\right] b_{l}^{k+1}\right) \\
& \quad+\frac{1}{2}\left(\left(\frac{u\left(x_{l+1}, t_{k+1}\right)-2 u\left(x_{l}, t_{k+1}\right)+u\left(x_{l-1}, t_{k+1}\right)}{(h)^{2}}\right)\right. \\
& \left.+\left(\frac{u\left(x_{l+1}, t_{k}\right)-2 u\left(x_{l}, t_{k}\right)+u\left(x_{l-1}, t_{k}\right)}{(h)^{2}}\right)\right) \\
& +\frac{1}{c_{0}^{2}} \frac{1}{2}\left(\left(\frac{u\left(x_{l+1}, t_{k+1}\right)-2 u\left(x_{l+1}, t_{k}\right)+u\left(x_{l+1}, t_{k-1}\right)}{(h)^{2}}\right)\right. \\
& \left.+\left(\frac{u\left(x_{l}, t_{k+1}\right)-2 u\left(x_{l}, t_{k}\right)+u\left(x_{l}, t_{k-1}\right)}{(h)^{2}}\right)\right)=E_{l}^{k+1} .
\end{aligned}
$$

From equations (3.1) and (3.4), we have

$$
\begin{aligned}
& \frac{\partial^{2} u\left(x_{l}, t_{k+1}\right)}{\partial x^{2}}+h^{2} V_{1}=\frac{1}{2}\left(\frac{\left(u\left(x_{l+1}, t_{k+1}\right)-2 u\left(x_{l}, t_{k+1}\right)+u\left(x_{l-1}, t_{k+1}\right)\right)}{h^{2}}\right. \\
& \left.+\frac{\left(u\left(x_{l+1}, t_{k}\right)-2 u\left(x_{l}, t_{k}\right)+u\left(x_{l-1}, t_{k}\right)\right)}{h^{2}}\right) \text {, } \\
& \frac{\partial^{\alpha_{l}^{k+1}} \Phi\left(r_{l}, t_{k+1}\right)}{\partial t^{\alpha_{l}^{k+1}}}+\sigma V_{2}=\frac{t^{-\rho_{l}^{k+1}}}{\Gamma\left(2-\rho_{l}^{k+1}\right)}\left(u\left(x_{l}, t_{k+1}\right)-u\left(x_{l}, t_{k}\right)\right. \\
& \left.+\sum_{j=1}^{k}\left[u\left(x_{l}, t_{k+1-j}\right)-u\left(x_{l}, t_{k-j}\right)\right] \lambda_{j}^{l, k}\right) \\
& \frac{\partial^{2} u\left(x_{l}, t_{k+1}\right)}{\partial t^{2}}+h^{2} V_{3}=\frac{1}{2}\left(\frac{\left(u\left(x_{l+1}, t_{k+1}\right)-2 u\left(x_{l+1}, t_{k}\right)+u\left(x_{l+1}, t_{k-1}\right)\right)}{h^{2}}\right. \\
& \left.+\frac{\left(u\left(x_{l}, t_{k+1}\right)-2 u\left(x_{l}, t_{k}\right)+u\left(x_{l}, t_{k-1}\right)\right)}{h^{2}}\right) .
\end{aligned}
$$

Then the expression $E_{l}^{k+1}$ can be in the following form:

$$
\begin{aligned}
E_{l}^{k+1}= & \frac{t^{-\rho_{l}^{k+1}}}{\Gamma\left(2-\rho_{l}^{k+1}\right)}\left(\frac{\partial^{\alpha_{l}^{k+1}} \Phi\left(r_{l}, t_{k+1}\right)}{\partial t^{\alpha_{l}^{k+1}}}+\frac{\partial^{2} u\left(x_{l}, t_{k+1}\right)}{\partial x^{2}}+\frac{\partial^{2} u\left(x_{l}, t_{k+1}\right)}{\partial t^{2}}\right) \\
& +V_{1} \sigma^{1+\rho_{l}^{k+1}}+V_{2} h^{2} \sigma^{\rho_{l}^{k}}+V_{3} h^{2} \sigma^{\rho_{l}^{k}} .
\end{aligned}
$$

From the above we have that

$$
R_{l}^{k+1} \leq K\left(\sigma^{1+\rho_{l}^{k+1}}+2 h^{2} \sigma_{l}^{\rho_{l}^{k}}\right)
$$

where $V_{1}, V_{2}, V_{3}$ and $K$ are constants. Taking into account the Caputo-type fractional derivative, the detailed error analysis on the above schemes can refer to the work in [31] and further work by [32]. 
Lemma $1\left\|\Omega^{k+1}\right\|_{\infty} \leq K\left(\sigma^{1+\rho_{l} k+1}+2 h^{2} \tau^{\alpha}{ }^{k}\right)\left(\Omega_{j}^{l, k+1}\right)^{-1}$ is true for $(k=0,1,2, \ldots, N-1)$, where $\left\|w^{k}\right\|_{\infty}=\max _{1 \leq l \leq M-1}\left(\Omega^{k}\right), K$ is a constant. In addition,

$$
\rho^{k+1}= \begin{cases}\min _{1 \leq l \leq M-1} \rho_{l}^{k+1} & \text { if } \tau<1 \\ \max _{1 \leq l \leq M-1} \rho_{l}^{k+1} & \text { if } \tau>1\end{cases}
$$

This can be achieved via the recurrence technique on the natural number $k$.

When $k=0$, we have the following:

$$
\begin{aligned}
& \left|\Omega_{l}^{1}\right| \leq\left|\Omega_{l}^{1}\right|\left(1+2 T_{l}^{1}-c_{0}^{2} T_{l}^{1}\right)+\left|\Omega_{l+1}^{1}\right|\left(c_{0}^{2} T_{l}^{1}+T_{l}^{1}\right)+\left|\Omega_{l-1}^{1}\right|\left(c_{0}^{2} T_{l}^{1}+T_{l}^{1}\right), \\
& \left|\Omega_{l}^{1}\left(1+2 T_{l}^{1}-c_{0}^{2} T_{l}^{1}\right)+\Omega_{l+1}^{1}\left(c_{0}^{2} T_{l}^{1}+T_{l}^{1}\right)+\Omega_{l-1}^{1}\left(c_{0}^{2} T_{l}^{1}+T_{l}^{1}\right)\right| \\
& \quad=\left|E_{l}^{1}\right| \leq K\left(\sigma^{1+\rho_{l} k+1}+2 h^{2} \sigma^{\rho_{l} k}\right)\left(\lambda_{j}^{l, k+1}\right)^{-1} .
\end{aligned}
$$

Now, suppose that $\left\|\Omega^{i+1}\right\|_{\infty} \leq K\left(\sigma^{1+\rho_{l} i+1}+2 h^{2} \sigma^{\rho l i}\right)\left(\lambda_{j}^{l, i+1}\right)^{-1}, i=1, \ldots, N-2$. Then

$$
\begin{aligned}
& \left|\Omega_{l}^{k+1}\right|=\left|\Omega_{l}^{k+1}\right|\left(1+2 T_{l}^{k+1}-c_{0}^{2} T_{l}^{k+1}\right)+\left|\Omega_{l+1}^{k+1}\right|\left(c_{0}^{2} T_{l}^{k+1}+T_{l}^{k+1}\right)+\left|\Omega_{l-1}^{k+1}\right|\left(c_{0}^{2} T_{l}^{k+1}+T_{l}^{k+1}\right), \\
& \left|\Omega_{l}^{k+1}\left(1+2 T_{l}^{k+1}-c_{0}^{2} T_{l}^{k+1}\right)+\Omega_{l+1}^{k+1}\left(c_{0}^{2} T_{l}^{k+1}+T_{l}^{k+1}\right)+\Omega_{l-1}^{k+1}\left(c_{0}^{2} T_{l}^{k+1}+T_{l}^{k+1}\right)\right| \\
& \quad=\left|R_{l}^{k+1}+\sum_{i=1}^{k}\left(\Omega_{l}^{k-i}\right) \lambda_{j}^{l, k+1}\right| \\
& \quad \leq\left|R_{l}^{k+1}\right|+\sum_{i=1}^{k}\left|\Omega_{l}^{k-i}\right| \lambda_{j}^{l, k+1} \\
& \quad \leq K\left(\sigma^{1+\rho_{l}^{k+1}}+2 h^{2} \sigma_{l}^{\rho_{l}^{k}}\right)+\sum_{i=1}^{k}\left\|\Omega_{l}^{k-i}\right\|_{\infty} \lambda_{j}^{l, k+1} \\
& \quad \leq K\left(\sigma^{1+\rho_{l}^{k+1}}+2 h^{2} \sigma_{l}^{\rho_{l}^{k}}\right)\left(\lambda_{j}^{l, k+1}+\lambda_{0}^{l, k+1}-\lambda_{j}^{l, k+1}\right)\left(\lambda_{j}^{l, k+1}\right)^{-1} \\
& \quad \leq V\left(\sigma^{1+\rho_{l}^{k+1}}+2 h^{2} \sigma_{l}^{\rho_{l}^{k}}\right)\left(\lambda_{0}^{l, k+1}\right)\left(\lambda_{j}^{l, k+1}\right)^{-1}, \\
& \left|\Omega_{l}^{1+k}\right| \leq V\left(\sigma^{1+\rho_{l}^{k+1}}+h^{2} \sigma_{l}^{\rho_{l}^{k}}\right)\left(\lambda_{j}^{l, k+1}\right)^{-1},
\end{aligned}
$$

which completes the proof.

Theorem 1 The Crank-Nicholson scheme is convergent, and there exists a positive constant K such that

$$
\left|u_{l}^{k}-u\left(x_{l}, t_{k}\right)\right| \leq K\left(\sigma+2 h^{2}\right), \quad l=1,2, \ldots, M-1, k=1,2, \ldots, N .
$$

The interested can find the solvability of the Crank-Nicholson scheme in the work done by [29]. Therefore the details of the proof will not be presented in this paper.

\section{Numerical simulation}

In this section we show the numerical simulation of the acoustic wave equation with variational-order derivative loss operator. In the simple case, we consider the variational order of equation (2.7) to be in the form of $\rho(x, t)=1+\sin (x t)$, where $(x, t) \in[-1,1] \times[0, t]$. The approximate solution of the main problem has been depicted in Figure 1 which is 


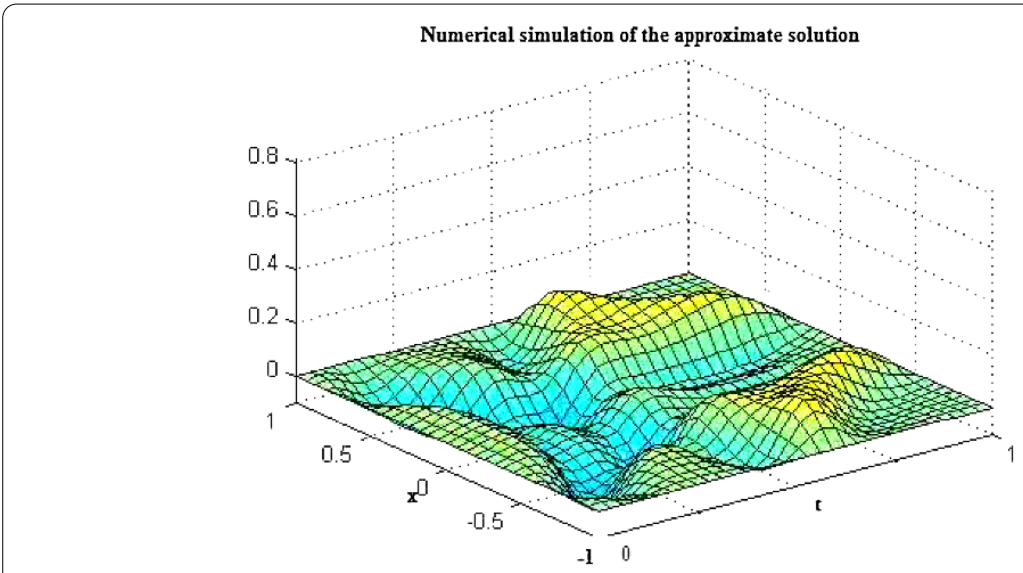

Figure 1 Numerical solution of the main problem for $\rho(x, t)=1+\sin (x t)$, where $(x, t) \in[-1,1] \times[0, t]$.

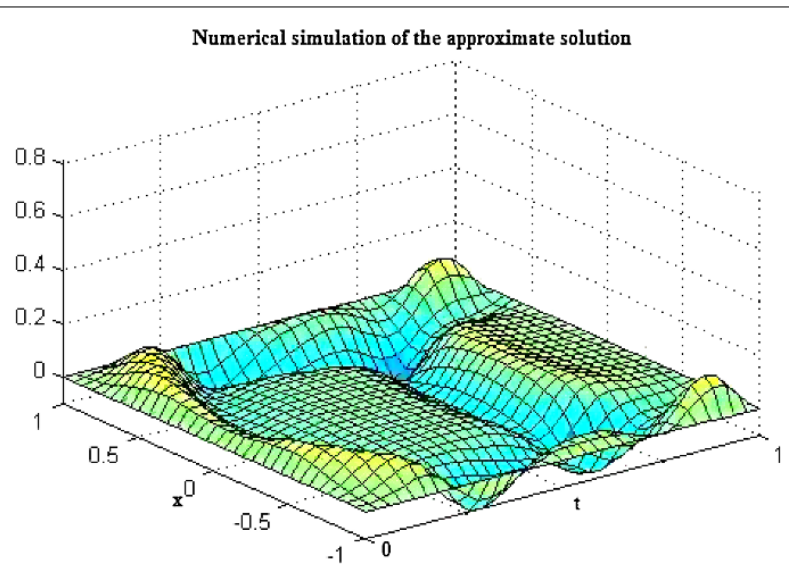

Figure 2 Numerical solution of the main problem $\rho(x, t)=1+\cos (x t)$, where $(x, t) \in[-1,1] \times[0, t]$.

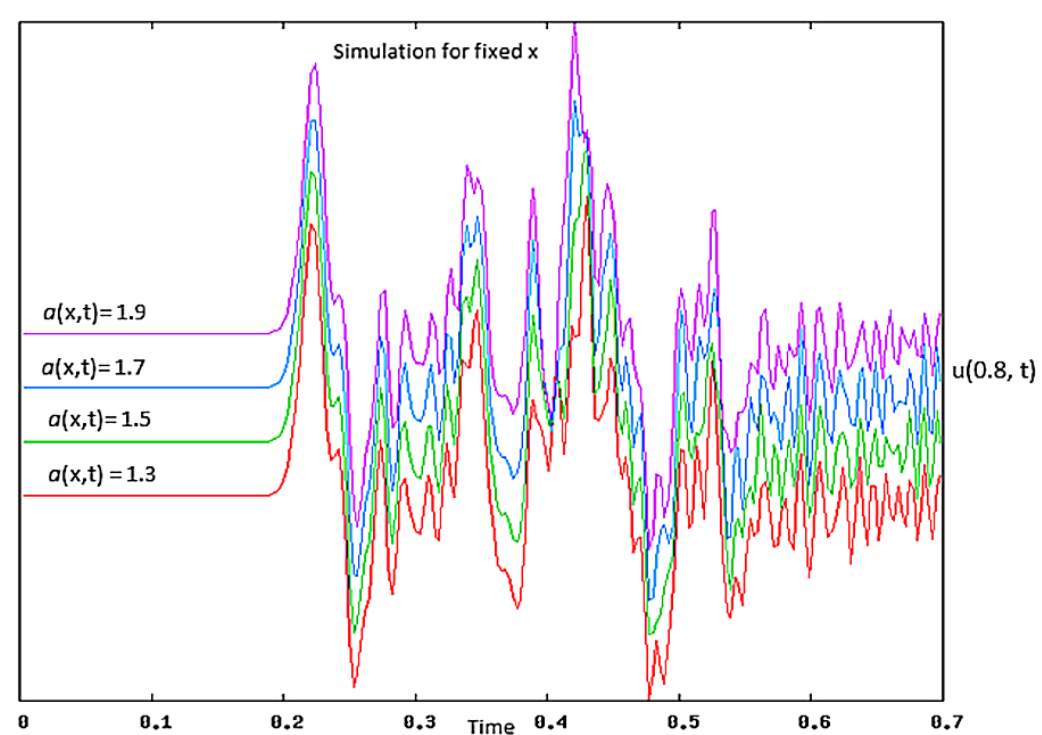

Figure 3 Simulation for a different constant fractional-order derivative. 
plotted in Mathematica. This figure shows the movement of the sound through the deformable medium. Figure 3 shows the simulation for a different constant fractional-order derivative as a function of $t$ for a fixed value of $x=0.8$.

In the simple case, we consider the variational order of equation (2.7) to be in the form of $\rho(x, t)=1+\cos (x t)$, where $(x, t) \in[-1,1] \times[0, t]$. The approximate solution of the main problem has been depicted in Figure 2, which is plotted in Mathematica. This figure shows the movement of the sound through the deformable medium.

\section{Conclusion}

The wave equation for viscous losses involving integer-order derivatives only leads to an attenuation which is proportional to the square of the frequency. This does not always reflect reality. The acoustic wave equation with loss operator was generalized using some approaches of variational calculus. Since the modified equation is difficult to solve analytically, we make use of the numerical scheme to solve this new equation. The numerical used in solving this new equation is the Crank-Nicholson scheme. The convergence and the stability of this scheme in this case were presented in details.

\section{Competing interests}

The author declares that they have no competing interests.

Received: 17 February 2013 Accepted: 24 May 2013 Published: 12 June 2013

\section{References}

1. Mainardi, F: The fundamental solutions for the fractional diffusion-wave equation. Appl. Math. Lett. 9, 23-28 (1996)

2. Schneider, WR, Wyss, W: Fractional diffusion and wave equations. J. Math. Phys. 30, 134-144 (1989)

3. Koh, C, Kelly, J: Application of fractional derivatives to seismic analysis of base-isolated models. Earthquake Eng Struct. Dyn. 19, 229-241 (1990)

4. Szabo, T: Time domain wave equations for lossy media obeying a frequency power law. J. Acoust. Soc. Am. 96, 491-500 (1994)

5. Buckingham, M: Theory of acoustic attenuation, dispersion, and pulse propagation in unconsolidated granular materials including marine sediments. J. Acoust. Soc. Am. 102, 2579-2596 (1997)

6. Norton, GV, Novarini, JC: Including dispersion and attenuation directly in the time domain for wave propagation in isotropic media. J. Acoust. Soc. Am. 113, 3024-3030 (2003)

7. Chen, W, Holm, S: Fractional Laplacian time-space models for linear and nonlinear lossy media exhibiting arbitrary frequency power-law dependency. J. Acoust. Soc. Am. 115, 1424-1430 (2004)

8. Duck, FA: Acoustic properties of tissue at ultrasonic frequencies. In: Physical Properties of Tissues - A Comprehensive Reference Book, chap. 4, pp. 98-108. Academic Press, San Diego (1990)

9. Kibblewhite, A: Attenuation of sound in marine sediments: a review with emphasis on new low-frequency data. J. Acoust. Soc. Am. 86, 716-738 (1989)

10. Wismer, M: Finite element analysis of broadband acoustic pulses through inhomogenous media with power law attenuation. J. Acoust. Soc. Am. 120, 3493-3502 (2006)

11. Holm, S, Sinkus, R: A unifying fractional wave equation for compressional and shear waves. J. Acoust. Soc. Am. 127, 542-548 (2010)

12. Treeby, B, Cox, B: Modeling power law absorption and dispersion for acoustic propagation using the fractional Laplacian. J. Acoust. Soc. Am. 127, 2741-2748 (2010)

13. Ochmann, M, Makarov, S: Representation of the absorption of nonlinear waves by fractional derivatives. J. Acoust. Soc. Am. 94, 3392-3399 (1993)

14. Liebler, M, Ginter, S, Dreyer, T, Riedlinger, R: Full wave modelling of therapeutic ultrasound: efficient time-domain implementation of the frequency power-law attenuation. J. Acoust. Soc. Am. 116, 2742-2750 (2004)

15. Tavakkoli, J, Cathignol, D, Souchon, R, Sapozhnikov, O: Modeling of pulsed finite amplitude focused sound beams in time domain. J. Acoust. Soc. Am. 104, 2061-2072 (1998)

16. Remenieras, J, Bou Matar, O, Labat, V, Patat, F: Time-domain modelling of nonlinear distortion of pulsed finite amplitude sound beams. Ultrasonics 38, 305-311 (2000)

17. Szabo, TL: Time domain wave equations for lossy media obeying a frequency power law. J. Acoust. Soc. Am. 96 491-500 (1994)

18. Szabo, TL, Wu, J: A model for longitudinal and shear wave propagation in viscoelastic media. J. Acoust. Soc. Am. 107, 2437-2446 (2000)

19. Chen, W, Holm, S: Fractional Laplacian time-space models for linear and nonlinear lossy media exhibiting arbitrary frequency power-law dependency. J. Acoust. Soc. Am. 115(4), 1424-1430 (2004). doi:10.1121/1.1646399

20. Wismer, MG: Finite element analysis of broad-band-acoustic pulses through inhomogenous media with power law attenuation. J. Acoust. Soc. Am. 120, 3493-3502 (2006) 
21. Zhang, Y: A finite difference method for fractional partial differential equation. Appl. Math. Comput. 215, 524-529 (2009)

22. Tadjeran, C, Meerschaert, MM, Scheffler, HP: A second order accurate numerical approximation for the fractional diffusion equation. J. Comput. Phys. 213, 205-213 (2006)

23. Meerschaert, MM, Tadjeran, C: Finite difference approximations for fractional advection dispersion equations. J. Comput. Appl. Math. 172, 65-77 (2004)

24. Yuste, SB, Acedo, L: An explicit finite difference method and a new Von Neumann-type stability analysis for fractional diffusion equations. SIAM J. Numer. Anal. 42, 1862-1874 (2005)

25. Podlubny, I, Chechkin, A, Skovranek, T, Chen, YQ, Vinagre Jara, BM: Matrix approach to discrete fractional calculus II: partial fractional differential equations. J. Comput. Phys. 228, 3137-3153 (2009)

26. Hanert, E: On the numerical solution of space-time fractional diffusion models. Comput. Fluids 46, 33-39 (2011)

27. Zhuang, $P, L i u, F, A n h, V$, Turner, I: Numerical methods for the variable-order fractional advection-dispersion equation with a nonlinear source term. SIAM J. Numer. Anal. 47, 1760-1781 (2009)

28. Lin, R, Liu, F, Anh, V, Turner, I: Stability and convergence of a new explicit finite-difference approximation for the variable-order nonlinear fractional diffusion equation. Appl. Math. Comput. 212, 435-445 (2009)

29. Crank, J, Nicolson, P: A practical method for numerical evaluation of solutions of partial differential equations of the heat conduction type. Proc. Camb. Philos. Soc. 43(1), 50-67 (1947). doi:10.1007/BF02127704

30. Langlands, TAM, Henry, BI: The accuracy and stability of an implicit solution method for the fractional diffusion equation. J. Comput. Phys. 205, 719-736 (2005)

31. Diethelm, K, Ford, NJ, Freed, AD: Detailed error analysis for a fractional Adams method. Numer. Algorithms 36, 31-52 (2004)

32. Li, CP, Tao, CX: On the fractional Adams method. Comput. Math. Appl. 58, 1573-1588 (2009)

doi:10.1186/1687-1847-2013-167

Cite this article as: Atangana: On the solution of an acoustic wave equation with variable-order derivative loss operator. Advances in Difference Equations 2013 2013:167.

\section{Submit your manuscript to a SpringerOpen ${ }^{\circ}$ journal and benefit from:}

- Convenient online submission

- Rigorous peer review

- Immediate publication on acceptance

- Open access: articles freely available online

- High visibility within the field

- Retaining the copyright to your article 